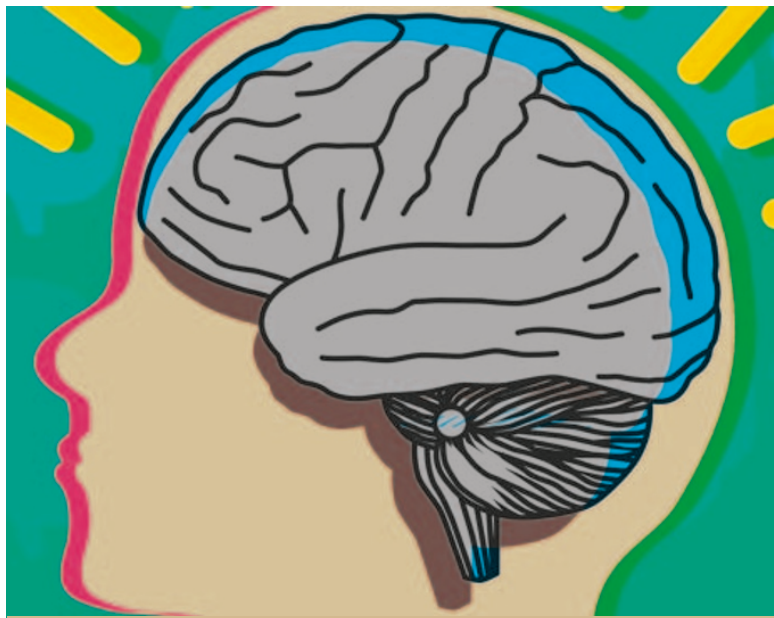

CANCER STEM CELLS

\section{BMPs on the brain}

Aggressive and recurrent glioblastomas have been shown to contain a fraction of multipotent cells with extensive selfrenewing properties that are likely to drive tumour growth. Angelo Vescovi and colleagues now show that bone morphogenetic proteins (BMPs) can block the growth of these brain-tumour-initiating cells (BTICs) and inhibit their ability to establish glioblastomas after transplantation.

BMPs direct normal brain stem cells towards an astroglial commitment, so the authors investigated whether they could also affect BTIC fate. They found that BMP4 and its cognate receptor are both expressed in glioblastoma cells, including the $\mathrm{CD}_{133^{+}}$subpopulation that is enriched in BTICs, and that the signalling pathway is functional, shown by the activation of the Smad signalling cascade. In vitro, BMP4 had a cytostatic effect on glioblastoma cells, reducing both their clonogenic index and growth rate, and inducing differentiation, as assessed by the detection of astroglial and neuronal markers. Remarkably, BMP4 reduced the subpopulation of glioblastoma cells that express $\mathrm{CD} 133^{+}$by almost $50 \%$, indicating that it targets the BTIC compartment by inducing these cells to differentiate.

The authors then tested whether BMP4 could also affect the tumorigenic BTIC pool in vivo. They pre-treated CD133+ glioblastoma cells with BMP4 and injected them into immunodeficient mice. Strikingly, BMP4-treated cells did not form invasive tumours, but only small delimited lesions, whereas all mice injected with untreated cells developed large tumours and died after just a few months. BMP4 was also delivered either during or after the injection of glioblastoma cells, and in both cases it blocked tumour development and significantly increased survival compared with control mice.

Therefore, BMP4 is a promising candidate for new strategies against malignant glioblastomas - inducing differentiation rather than killing the tumour-initiating cells.

\title{
Mysteries of addiction
}

Although several mutations and chromosomal aberrations typically contribute to the transformation of a cell, the inactivation of a single oncoprotein is sometimes enough to cause rapid apoptosis - it is as though the cancer cell has become dependent on a single oncogenic pathway that is not required in its noncancerous counterpart. Although such 'oncogene addiction' is important (and useful) for the development of targeted therapies, the underlying mechanism is not clear. Now, Jeffrey Settleman and colleagues propose a new mechanism to explain the phenomenon, which they feel is more accurately described as 'oncogenic shock'.

The researchers propose (as published in Clinical Cancer Research) that the oncoproteins that maintain a cancer cell have both pro-survival and pro-apoptotic effects on a cell. They suggest that when the activity of a single oncoprotein is disrupted, the pro-survival signals are attenuated faster than pro-apoptotic signals, and that this is enough to temporarily shift the balance in favour of apoptosis.

Now, in Cancer Cell, the authors have empirical evidence to support this idea. They measured the pro-apoptotic and pro-survival factors in several cell culture models - a cell line dependent on the BCR-ABL oncoprotein, a cell line dependent on mutationally activated epidermal growth factor receptor (EGFR), and a non-small cell lung cancer cell line with an activating mutation within EGFR. When the oncogenes were inactivated, the levels of pro-survival factors (Akt, signal transducer and activator of transcription 5 and extracellular-signal regulated kinase 1 and 2) quickly fell, whereas the levels of a pro-apoptotic factor (the activated form of the p38 mitogen-activated protein kinase (phospho-p38)) accumulated, followed by rapid apoptosis.

The authors then delved more deeply into why phospho-p38 lingers for longer than the pro-survival factors. Their results indicate that the accumulation of phospho-p38 could be delayed owing to the need for a reduction of phosphatase activity before phosphorylation of p38 can occur, leading the researchers to suggest that it is these phosphatases that might orchestrate the timing of signal attenuation.

Understanding the mechanisms of oncogenic shock has important clinical implications. Therapies that target oncoproteins might be augmented by additional downstream factors that further shift the phosphatase-mediated balance towards apoptosis. The authors also discuss ways of providing more opportunities to produce this transient imbalance in pro-survival and pro-apoptotic signals during treatment, and they suggest that several 'on-off' cycles of kinase inhibitors might be effective.

Jenny Bangham

ORIGINAL RESEARCH PAPER Sharma, S. V.et al. A common signalling cascade may underlie 'addiction' to the Src, BCR-ABL, and EGF receptor oncogenes. Cancer Cell 10, 425-435 (2006)

FURTHER READING Sharma, S. V, Fischbach, M A., Haber, D. A. \& Settleman, J. 'Oncogenic shock' explaining oncogene addiction through differential signal attenuation. Clin. Cancer Res. 